\title{
A robust approach for analysing dispersion of elastic waves in an orthotropic cylindrical shell
}

\author{
J. Kaplunov ${ }^{\mathrm{a}}$, A. Nobili ${ }^{\mathrm{b}, *}$ \\ ${ }^{a}$ School of Computing and Mathematics, Keele University, Keele, Staffordshire, ST5 5BG, \\ $U K$ \\ ${ }^{b}$ Università degli Studi di Modena e Reggio Emilia, Dipartimento di Ingegneria Enzo \\ Ferrari, via Vivarelli 10, 41125 Modena, Italy
}

\begin{abstract}
Dispersion of elastic waves in a thin orthotropic cylindrical shell is considered, within the framework of classical 2D Kirchhoff-Love theory. In contrast to direct multi-parametric analysis of the lowest propagating modes, an alternative robust approach is proposed that simply requires evaluation of the evanescent modes (quasi-static edge effect), which, at leading order, do not depend on vibration frequency. A shortened dispersion relation for the propagating modes is then derived by polynomial division and its accuracy is numerically tested against the full Kirchhoff-Love dispersion relation. It is shown that the same shortened relation may be also obtained from a refined dynamic version of the semi-membrane theory for cylindrical shells. The presented results may be relevant for modelling various types of nanotubes which, according to the latest experimental findings, possess strong material anisotropy.
\end{abstract}

Keywords: Thin-shell, vibration, anisotropy, nanotube, elastic, low-frequency

\section{Introduction}

A fresh interest in mechanics of thin elastic shells has emerged in the last few decades due to advanced applications in nanotechnology, e.g. see $[1,2,3,4,5]$. In particular, dynamic analysis of elongated carbon nanotubes inspires derivation

${ }^{*}$ Corresponding author 
of novel equations governing the low-frequency near-cutoff regime. The original treatment in [6], for an isotropic cylindrical shell, is based on the geometric hypotheses underlying the approximate semi-membrane theory for shells, e.g. see $[7,8]$, assuming the absence of shear and circumferential elongation of the mid-surface. Later on, this formulation has been refined using an asymptotic methodology [9].

This paper is motivated by a clear demand for taking into consideration nanotube anisotropy, which has been observed in experimental measurements. For instance, in [10] multi-walled carbon nanotube mechanical properties are determined through atomic force microscopy and it is found that for ordered arcgrown tubes the radial elastic constant is roughly one third of the axial constant. Indeed, this result is well expected because of strong anisotropy of graphite [11]. Furthermore, experimental data are consistent with a conventional forceconstant model $[12,10]$. Recent investigations indicates that also single-walled carbon nanotubes exhibit strong chirality-induced anisotropy [13, 14]. Besides carbon nanotubes, oxidic nanotubes also demonstrate anisotropic properties [15].

Similarly to [9], focus is here set on the lowest propagating modes, which are strongly affected by shell thickness, vibration frequency, and also by circumferential wavenumber. The pretty sophisticated multi-parametric nature of these modes makes rather cumbersome their direct derivation from the full dispersion relation for an orthotropic shell, within the framework of the classical KirchhoffLove theory. At the same time, another solution set of the full equation corresponds to well-behaved evanescent modes forming the so-called edge effect, e.g. see $[16,7,8]$. It is crucial that, at leading order, the evanescent modes do not depend on vibration frequency and wavenumber, and thus they can be easily evaluated. This observation suggests a robust implicit approach for establishing a shortened dispersion relation for the propagating modes. First, we obtain an approximate equation for the evanescent modes. Then, using the latter, we extract the sought-for shortened dispersion relation by elementary polynomial division of the full equation. The shortened relation greatly facilitates a qual- 
itative insight into the peculiarities of dispersion of low-frequency waves. We remark that this dispersion relation is an example of a near cutoff asymptotic expansion over the low-frequency range. It is interesting that the near cutoff expansions for thin elastic structures have been previously obtained only over the high-frequency range, in the vicinity of thickness resonance frequencies, also including the effects of anisotropy [17] and environment [18, 19].

The paper is organised as follows. In Sec.2 we obtain the Koiter-Sanders version of the Kirchhoff-Love equations for an orthotropic cylindrical shell, e.g. see [20], starting from standard variational arguments. In the next section, we deduce the associated dispersion relation along with the formulae for eigenforms and cutoff frequencies. It is shown that the lowest cutoff frequency is asymptotically small for a thin shell. Sec.4 deals with the edge effect and a fourth-order expansion is here obtained for the related evanescent solutions. The results of this section are exploited in Sec.5 to derive a dispersion relation for the propagating modes. As a particular example, the case of an isotropic material is presented. The asymptotic cancellations, typical of the near cutoff behaviour, are thoroughly investigated. Numerical results validating the shortened dispersion relation are demonstrated. Amended hypotheses in the aforementioned semi-membrane shell theory are formulated on the basis of two-term expansions for the eigenforms. As it might be expected, the variationally reduced PDE following from the amended hypotheses supports the same shortened dispersion relation as that developed in the previous section. This PDE seemingly has a potential to be a useful model for tackling various modern problems in anisotropic shell dynamics.

\section{Variational derivation of the equations of motion}

We consider linear vibrations of a thin orthotropic circular cylindrical shell starting from the Sanders-Koiter version of the classical Kirchhoff-Love the-

ory, e.g. see [20]. Accordingly, the Lagrangian density function, in light of the 
assumptions $\sigma_{z z}=\sigma_{x z}=\sigma_{x \theta}=0$, is given by

$$
\mathcal{L}=\frac{1}{2} \rho\left[\left(\partial_{t} u\right)^{2}+\left(\partial_{t} v\right)^{2}+\left(\partial_{t} w\right)^{2}\right]-\frac{1}{2}\left(\sigma_{x x} \epsilon_{x x}+\sigma_{x \theta} \epsilon_{x \theta}+\sigma_{\theta \theta} \epsilon_{\theta \theta}\right),
$$

where $x, z$ and $\theta$ are, respectively, the axial, the radial and the azimuthal coordinates in a cylindrical reference system and $\partial_{t}$ is shorthand for $\partial / \partial t$. Here, $u, v$ and $w$ describe the longitudinal, circumferential and radial mid-surface displacements, $\rho$ is the mass density, $\boldsymbol{\epsilon}$ and $\boldsymbol{\sigma}$ are the linear strain and stress tensors, respectively. Clearly, the first term in (1) is the kinetic energy density, while the second term represents the elastic stored energy.

Let $L$ be a characteristic length (for instance, a typical wavelength), $h$ the uniform shell thickness and $R$ the shell mid-surface radius. Then, the action integral is

$$
A=\int_{t_{1}}^{t_{2}} \int_{0}^{L} \int_{0}^{2 \pi} \int_{-h / 2}^{h / 2} \mathcal{L} \mathrm{d} z \mathrm{~d} \theta \mathrm{d} x \mathrm{~d} t .
$$

The strain tensor is assumed to vary linearly through the thickness [20, $§ 1.3 .4]$

$$
\epsilon_{x x}=\epsilon_{0 x}+z k_{x}, \quad \epsilon_{x \theta}=\epsilon_{0 x \theta}+z k_{x \theta}, \quad \epsilon_{\theta \theta}=\epsilon_{0 \theta}+z k_{\theta},
$$

where the mid-surface strains are defined as [21, Eq.(3.18)]

$$
\epsilon_{0 x}=L^{-1} \partial_{\xi} u, \quad \epsilon_{0 \theta}=R^{-1}\left(\partial_{\theta} v+w\right), \quad \epsilon_{0 x \theta}=R^{-1} \partial_{\theta} u+L^{-1} \partial_{\xi} v
$$

while, for the mid-surface curvatures and torsion, we have $[20, \S 1.3 .8]$

$$
\begin{aligned}
k_{x} & =-L^{-2} \partial_{\xi \xi}^{2} w, \\
k_{\theta} & =R^{-2}\left(\partial_{\theta} v-\partial_{\theta \theta}^{2} w\right), \\
k_{x \theta} & =(L R)^{-1}\left(-2 \partial_{\xi \theta}^{2} w+\frac{3}{2} \partial_{\xi} v-\frac{1}{2} \partial_{\theta} u\right) .
\end{aligned}
$$

Hereinafter, the dimensionless axial coordinate $\xi=x / L$ is introduced. The constitutive equations will be assumed in the form

$$
\left[\begin{array}{c}
\sigma_{x x} \\
\sigma_{\theta \theta} \\
\sigma_{x \theta}
\end{array}\right]=\mathbf{C}\left[\begin{array}{c}
\epsilon_{x x} \\
\epsilon_{\theta \theta} \\
\epsilon_{x \theta}
\end{array}\right], \quad \mathbf{C}=\left[\begin{array}{ccc}
c_{11} & c_{12} & 0 \\
c_{12} & c_{22} & 0 \\
0 & 0 & c_{66}
\end{array}\right],
$$


which is valid for cylindrical orthotropic materials [21]. The shell material is homogeneous, whence the material parameters $c_{11}, c_{22}, c_{12}$ and $c_{66}$ are constant. Besides, it is demanded that

$$
\left(c_{11}, c_{22}, c_{66}, c_{11} c_{22}-c_{12}^{2}\right)>0
$$

which warrants that the stiffness matrix $\mathbf{C}$ is positive definite. The constitutive parameters $c_{11}, c_{22}, c_{12}$ and $c_{66}$ are often expressed in terms of the technical material constants $E_{1}, E_{2}, \nu_{12}, \nu_{21}$ and $G$ provided that the reciprocal relation $\nu_{12} E_{2}=\nu_{21} E_{1}$ holds $[22, \S 2.5]$. For instance, for high-modulus graphite-epoxy unidirectional composite materials, we have [22, Table 2-3b]

$$
E_{1}=207 \mathrm{GPa}, \quad E_{2}=5 \mathrm{GPa}, \quad G=c_{66}=2.6 \mathrm{GPa}, \quad \nu_{12}=0.25,
$$

and therefore

$$
c_{11}=207.31 \mathrm{GPa}, \quad c_{22}=5.00 \mathrm{GPa}, \quad c_{12}=1.25 \mathrm{GPa} .
$$

As usual, density depends on the reinforcement to matrix volume fraction; for instance, $\rho=970 \mathrm{~kg} / \mathrm{m}^{3}$ for GY-70-HYE1534. For transversely isotropic materials (also named polar anisotropic), such as compression-annealed pyrolytic graphite [11], it is further

$$
c_{11}=c_{22} \quad \text { and } \quad 2 c_{66}=c_{11}-c_{12} .
$$

In particular, the isotropic case is retrieved by taking

$$
c_{11}=c_{22}=\lambda+2 G, \quad c_{12}=\lambda, \quad c_{66}=G
$$

where $\lambda=\nu E /\left(1-\nu^{2}\right)$ and $G=E /(2+2 \nu)$ are Lamé constants, which relate to $E=E_{1}=E_{2}$, Young's modulus, and $\nu=\nu_{12}=\nu_{21}$, Poisson's ratio.

Let us define the dimensionless time $\tau=c_{L} t / R$, where $c_{L}=\sqrt{c_{11} / \rho}$ is the speed of longitudinal waves in orthotropic media $[23, \S 7]$. In addition to a characteristic time scale, shell dynamics is governed by two geometric parameters, namely the inverse aspect ratio $\alpha=R / L$ and the relative thickness $\beta=h / R$. According to the usual restrictions of the 2D shell theory, we take

$$
\beta(1+\alpha) \ll 1,
$$


meaning that $h \ll \min (R, L)$.

On substituting Eqs.(3-6) into the action integral (2), performing the throughthe-thickness integration and varying, we find the Euler-Lagrange (E-L) equations for an orthotropic circular cylindrical shell within the linear Koiter-Sanders framework,

$$
\begin{aligned}
& \frac{\left(\beta^{2}+48\right) c_{66}}{48 c_{11}} \partial_{\theta \theta}^{2} u+\alpha \frac{16 c_{12}-\left(\beta^{2}-16\right) c_{66}}{16 c_{11}} \partial_{\xi \theta}^{2} u+\alpha \beta^{2} \frac{c_{66}}{12 c_{11}} \partial_{\xi \theta \theta}^{3} w \\
& +\alpha \frac{c_{12}}{c_{11}} \partial_{\xi} w+\alpha^{2} \partial_{\xi \xi}^{2} u-\partial_{\tau \tau}^{2} u=0 \\
& -\alpha \frac{\left(\beta^{2}-16\right) c_{66}-16 c_{12}}{16 c_{11}} \partial_{\xi \theta}^{2} u+\alpha^{2} \frac{\left(3 \beta^{2}+16\right) c_{66}}{16 c_{11}} \partial_{\xi \xi}^{2} v+\frac{c_{22}\left(\beta^{2}+12\right)}{12 c_{11}} \partial_{\theta \theta}^{2} v \\
& -\alpha^{2} \beta^{2} \frac{c_{12}+3 c_{66}}{12 c_{11}} \partial_{\xi \xi \theta}^{3} w-\beta^{2} \frac{c_{22}}{12 c_{11}} \partial_{\theta \theta \theta}^{3} w+\frac{c_{22}}{c_{11}} \partial_{\theta} w-\partial_{\tau \tau}^{2} v=0 \\
& -\alpha \beta^{2} \frac{c_{66}}{12 c_{11}} \partial_{\xi \theta \theta}^{3} u-\alpha \frac{c_{12}}{c_{11}} \partial_{\xi} u+\alpha^{2} \beta^{2} \frac{c_{12}+3 c_{66}}{12 c_{11}} \partial_{\xi \xi \theta}^{3} v+\beta^{2} \frac{c_{22}}{12 c_{11}} \partial_{\theta \theta \theta}^{3} v-\frac{c_{22}}{c_{11}} \partial_{\theta} v \\
& -\alpha^{2} \beta^{2} \frac{c_{12}+2 c_{66}}{6 c_{11}} \partial_{\xi \xi \theta \theta}^{4} w-\beta^{2} \frac{c_{22}}{12 c_{11}} \partial_{\theta \theta \theta \theta}^{4} w-\frac{c_{22}}{c_{11}} w-\frac{1}{12} \alpha^{4} \beta^{2} \partial_{\xi \xi \xi \xi}^{4} w-\partial_{\tau \tau}^{2} w=0
\end{aligned}
$$

On separating the circumferential co-ordinate through letting

$$
u=u_{n}(\xi, \tau) \sin (n \theta), \quad v=v_{n}(\xi, \tau) \cos (n \theta), \quad w=w_{n}(\xi, \tau) \sin (n \theta), \quad n \in \mathbb{N},
$$

the system of PDEs (11) reduces to

$$
\begin{aligned}
& -n^{2} \frac{c_{66}\left(\beta^{2}+48\right)}{48 c_{11}} u_{n}+\alpha \frac{12 c_{12}-\beta^{2} c_{66} n^{2}}{12 c_{11}} \partial_{\xi} w_{n} \\
& -\alpha n \frac{c_{66}\left(\beta^{2}-16\right)-16 c_{12}}{16 c_{11}} \partial_{\xi} v_{n}+\alpha^{2} \partial_{\xi \xi}^{2} u_{n}-\partial_{\tau \tau}^{2} u_{n}=0 \\
& -n^{2} \frac{c_{22}\left(\beta^{2}+12\right)}{12 c_{11}} v_{n}-n \frac{c_{22}\left(\beta^{2} n^{2}+12\right)}{12 c_{11}} w_{n}+\alpha^{2} \frac{c_{66}\left(3 \beta^{2}+16\right)}{16 c_{11}} \partial_{\xi \xi}^{2} v_{n} \\
& -\alpha n \frac{16 c_{12}-\left(\beta^{2}-16\right) c_{66}}{16 c_{11}} \partial_{\xi} u_{n}+\alpha^{2} \beta^{2} n \frac{c_{12}+3 c_{66}}{12 c_{11}} \partial_{\xi \xi}^{2} w_{n}-\partial_{\tau \tau}^{2} v_{n}=0 \\
& -\frac{c_{22}\left(\beta^{2} n^{4}+12\right)}{12 c_{11}} w_{n}+\alpha \frac{\beta^{2} c_{66} n^{2}-12 c_{12}}{12 c_{11}} \partial_{\xi} u_{n} \\
& -n \frac{c_{22}\left(\beta^{2} n^{2}+12\right)}{12 c_{11}} v_{n}+\alpha^{2} \beta^{2} n^{2} \frac{c_{12}+2 c_{66}}{6 c_{11}} \partial_{\xi \xi}^{2} w_{n} \\
& +\alpha^{2} \beta^{2} n \frac{c_{12}+3 c_{66}}{12 c_{11}} \partial_{\xi \xi}^{2} v_{n}-\frac{1}{12} \alpha^{4} \beta^{2} \partial_{\xi \xi \xi \xi}^{4} w_{n}-\partial_{\tau \tau}^{2} w_{n}=0 .
\end{aligned}
$$




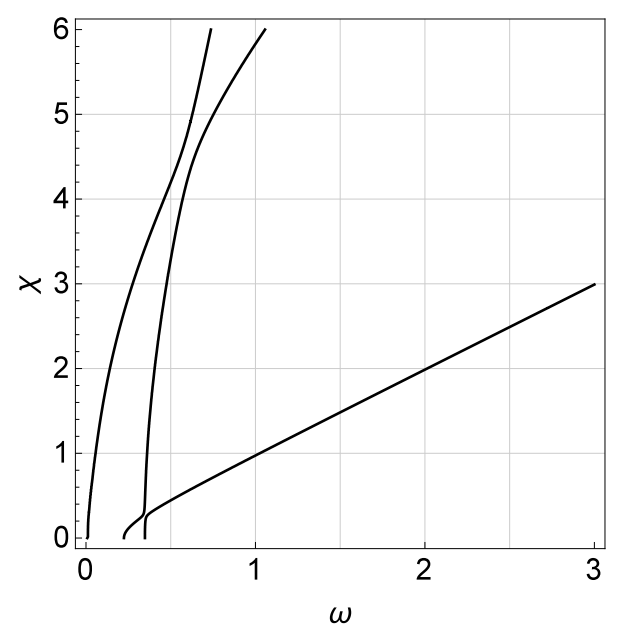

Figure 1: Three-branch dispersion curve for a graphite-epoxy composite shell (parameters as in $(8)$ and $n=2, \beta=0.1$ )

\section{Dispersion relation}

Assuming a travelling-wave solution

$$
\left\{u_{n}(\xi), v_{n}(\xi), w_{n}(\xi)\right\}=\left\{U_{n}, V_{n}, W_{n}\right\} \exp \left[\imath\left(\frac{\chi}{\alpha} \xi-\omega \tau\right)\right]
$$

we obtain the dispersion relation, in the form of a bi-quartic in $\chi$, i.e. a fourthdegree polynomial in $k=\chi^{2}$,

$$
a_{4} k^{4}+a_{3} k^{3}+\beta^{-2} a_{2} k^{2}+a_{1} k+a_{0}=0
$$

Exact expressions for the coefficients $a_{i}$, as well as for the eigenforms, are presented in Appendix A and Appendix B, respectively. We remark that the associated dispersion relation in 3D elasticity can be found, for example, in [24]. In addition, we also mention the recent paper [25] studying in-plane vibrations of a cylindrical isotropic layer. As expected, the coefficients $a_{i}$ in the dispersion relation (14) are independent of $\alpha$. Fig.1 shows the three branches of the dispersion relation for $n=2$.

The solution of the equation

$$
a_{0}=0
$$




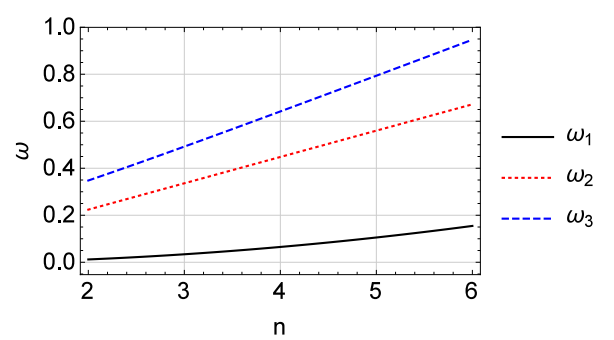

Figure 2: Cutoff frequencies for the three-branch dispersion curve, $\omega_{1}<\omega_{2}<\omega_{3}$, for a graphite-epoxy composite shell (parameters as in (8) and $\beta=0.1$ ) vs. circumferential wavenumber $n$

lends the expression for the three cutoff frequencies $\omega=\omega_{1,2,3}$, where

$$
\begin{aligned}
\omega_{1,3}^{2}=\frac{c_{22}}{24 c_{11}} & \left\{\left(n^{2}+1\right)\left(\beta^{2} n^{2}+12\right)\right. \\
& \left. \pm \sqrt{144\left(n^{2}+1\right)^{2}+\beta^{4} n^{4}\left(n^{2}+1\right)^{2}-24 \beta^{2} n^{2}\left(n^{4}-6 n^{2}+1\right)}\right\}
\end{aligned}
$$

and

$$
\omega_{2}^{2}=n^{2}\left(1+\frac{1}{48} \beta^{2}\right) \frac{c_{66}}{c_{11}} .
$$

It is easy to show that $\omega_{1}$ is the lowest cutoff frequency, as it can be rewritten as

$$
\begin{aligned}
& \omega_{1}^{2}=\beta^{2} \frac{2 c_{22} n^{2}\left(n^{2}-1\right)^{2}}{c_{11}}\left[\left(n^{2}+1\right)\left(\beta^{2} n^{2}+12\right)\right. \\
& \left.\quad+\sqrt{\left(n^{2}+1\right)^{2}\left(\beta^{4} n^{4}+144\right)-24 \beta^{2} n^{2}\left(n^{4}-6 n^{2}+1\right)}\right]^{-1}
\end{aligned}
$$

whence it is deduced that $\omega_{1} \sim \beta$ in contrast to $\omega_{2,3} \sim 1$. Fig.2 plots the cutoff frequencies $\omega_{1}<\omega_{2}<\omega_{3}$ as a function of the circular wavenumber $n$. Analysis of the lowest dispersion curve, corresponding to this cutoff frequency, is of substantial interest for modern applications, e.g. see [6], and, accordingly, it is the main focus of the paper.

The coefficients $a_{i}$ in the bi-quartic equation (14) depend on the three problem's parameters, namely $\beta, \omega$ and also $n$. In the low-frequency range $\omega \ll 1$, the analysis developed for an isotropic cylindrical shell [9] suggests that two roots of the dispersion relation, corresponding to propagating vibration modes, demonstrate a rather sophisticated three-parameter dependence. At the same time, 
the two other roots of this equation are usually related to evanescent modes, corresponding to the so-called edge effect (see, for example, $[7,8]$ ). At leading order, the evanescent modes do not depend both on frequency (whence they are referred to as being quasi-static) and also on the circumferential wavenumber $n$. This observation suggests a scheme which first demands evaluating the roots associated with the edge effect and then it proceeds to finding the propagatingmode roots of interest by factoring these evanescent modes out of the dispersion relation (14).

\section{Edge effect}

Similarly to an isotropic shell $[7,8]$, we may expect the leading order equation of the edge effect in the form

$$
\frac{\mathrm{d}^{4} w_{n}}{\mathrm{~d} \zeta^{4}}+\kappa w_{n}=0,
$$

where $w_{n}$ is the shell mid-surface displacement, $\zeta=\xi /(\alpha \sqrt{\beta})$ and $\kappa$ is a positive constant. This equation is sometimes referred to as bending boundary layer, see $[26, \S 10.3]$. It is clear from Eq.(17) that the roots of the dispersion relation (14), associated with the edge effect, support a quasi-static behaviour.

Let us now introduce the scalings

$$
\omega=\beta \Omega, \quad k=\frac{\mu}{\beta}
$$

into the equation (14) to get

$$
a_{4} \mu^{4}+\beta a_{3} \mu^{3}+a_{2} \mu^{2}+\beta^{3} a_{1} \mu+\beta^{4} a_{0}=0 .
$$

The coefficients $a_{i}, i \in\{1,2,3,4\}$ may be written as an expansion in $\beta$

$$
a_{i}=a_{i}^{(0)}+\beta^{2} a_{i}^{(2)}+\beta^{4} a_{i}^{(4)}+\beta^{6} a_{i}^{(6)}
$$

and they are defined as to be of order unity, see Appendix A.

We seek a solution of the dispersion relation (18) in the form of the asymptotic expansion

$$
\mu=\mu^{(0)}+\beta \mu^{(1)}+\beta^{2} \mu^{(2)}+\beta^{3} \mu^{(3)}+\ldots
$$


also assuming $n \ll \beta^{-1 / 2}$. Dealing with the leading order problem

$$
a_{4}^{(0)} \mu^{(0)^{2}}+a_{2}^{(0)}=0
$$

leads to the static solutions (evanescent modes)

$$
\mu_{1,2}^{(0)}= \pm \imath m_{0}, \quad m_{0}=\sqrt{\frac{a_{2}^{(0)}}{a_{4}^{(0)}}}=2 \sqrt{3} \frac{\sqrt{c_{11} c_{22}-c_{12}^{2}}}{c_{11}},
$$

wherein $m_{0}>0$ in light of the constraints (7). Proceeding to the next order, we have the expression

$$
\mu_{1,2}^{(1)}=\frac{a_{3}^{(0)} m_{0}^{2}}{2\left(a_{2}^{(0)}-2 a_{4}^{(0)} m_{0}^{2}\right)}
$$

which lends the yet static correction

$$
\mu_{1,2}^{(1)}=m_{1}=-n^{2} \frac{4 c_{66}^{2}+c_{11} c_{22}-c_{12}^{2}}{2 c_{11} c_{66}}<0,
$$

independently of the choice of $\mu_{1}^{(0)}$ or $\mu_{2}^{(0)}$. Similarly, proceeding to the next order, we get the first dynamic contribution

$$
\mu_{1,2}^{(2)}= \pm \imath \frac{a_{2}^{(0)} m_{1}^{2}-m_{0}^{2}\left[a_{2}^{(2)}+3 m_{1}\left(a_{3}^{(0)}+2 a_{4}^{(0)} m_{1}\right)\right]+a_{4}^{(2)} m_{0}^{4}}{2 m_{0}\left(2 a_{4}^{(0)} m_{0}^{2}-a_{2}^{(0)}\right)}
$$

which reads

$$
\mu_{1,2}^{(2)}= \pm \imath m_{2}
$$

where $m_{2}$ is given by

$$
\begin{aligned}
m_{2}= & \left\{-c_{11}^{2}\left(c_{22}^{2} n^{4}+48 c_{66}^{2} \Omega^{2}\right)-c_{12}^{4} n^{4}-16 c_{66}^{4} n^{4}-8 c_{66} c_{12}^{3} n^{2}\left(n^{2}-1\right)\right. \\
& +8 c_{66}\left(c_{11} c_{22}-4 c_{66}^{2}\right) c_{12} n^{2}\left(n^{2}-1\right)+8 c_{11} c_{22} c_{66}^{2} n^{2}\left(2 n^{2}-3\right) \\
& \left.+c_{12}^{2}\left(2 c_{11} c_{22} n^{4}-8 c_{66}^{2} n^{2}\left(3 n^{2}-4\right)\right)\right\} /\left(16 \sqrt{3} c_{11} c_{66}^{2} \sqrt{c_{11} c_{22}-c_{12}^{2}}\right) .
\end{aligned}
$$

Finally,

$$
\begin{array}{r}
\mu_{1,2}^{(3)}=\frac{a_{1}^{(0)}+2 a_{2}^{(2)} m_{1}+3 a_{3}^{(0)} m_{1}^{2}-m_{0}^{2}\left(a_{3}^{(2)}+4 a_{4}^{(2)} m_{1}\right)+4 a_{4}^{(0)} m_{1}^{3}}{4 a_{4}^{(0)} m_{0}^{2}-2 a_{2}^{(0)}} \\
-m_{2} \frac{3 m_{0}^{2}\left(a_{3}^{(0)}+4 a_{4}^{(0)} m_{1}\right)-2 a_{2}^{(0)} m_{1}}{4 a_{4}^{(0)} m_{0}^{3}-2 a_{2}^{(0)} m_{0}}
\end{array}
$$


whence

$$
\mu_{1,2}^{(3)}=m_{3}
$$

with

$$
\begin{gathered}
m_{3}=\left\{96 c_{11}^{2} c_{66} c_{12} n^{2} \Omega^{2}+5 c_{12}^{4} n^{2}-c_{12}^{2}\left[-48 c_{11}^{2} n^{2} \Omega^{2}+20 c_{66}^{2} n^{2}\right.\right. \\
\left.+2 c_{11}\left(c_{22}\left(2 n^{4}-4 n^{2}+7\right) n^{2}+24 c_{66} \Omega^{2}\right)\right] \\
\left.-c_{11} c_{22} n^{2}\left(c_{22} c_{11}\left(-4 n^{4}+8 n^{2}-9\right)-4 c_{66}^{2}\left(4 n^{4}-8 n^{2}+9\right)+48 c_{11}^{2} \Omega^{2}\right)\right\} \\
/\left(96 c_{11}\left(c_{11} c_{22}-c_{12}^{2}\right) c_{66}\right) .
\end{gathered}
$$

As a result, we obtain the asymptotic expression of the evanescent modes

$$
k_{1,2}=\beta^{-1}\left( \pm \imath m_{0}+\beta m_{1} \pm \imath \beta^{2} m_{2}+\beta^{3} m_{3}+\ldots\right),
$$

together with the expression for the sum and the product of the roots, respectively

$$
p_{1}=k_{1}+k_{2}=2 m_{1}+2 \beta^{2} m_{3}+\ldots,
$$

and

$$
q_{1}=k_{1} k_{2}=\beta^{-2} m_{0}^{2}+m_{1}^{2}-2 m_{0} m_{2}+\ldots
$$

It is observed that, although the edge effect roots $k_{1}$ and $k_{2}$ are large (precisely, they are of order $\beta^{-1}$ ), their sum $p_{1}$ is of order unity, owing to cancellation of the odd order terms. Here, for the following derivations, two terms are retained in the expansions for $p_{1}$ and $q_{1}$ and, to this end, we keep four terms in the root expansion (20).

\section{Shortened dispersion relation for propagating modes}

The dispersion relation (14) may now be rewritten as the product of two second degree polynomials in $k$, namely

$$
\left(k^{2}-p_{1} k+q_{1}\right)\left(k^{2}-p_{2} k+q_{2}\right)=0,
$$


which allows determining the sought-for quantities $p_{2}$ and $q_{2}$, associated with propagating vibration modes, by polynomial division

$$
\begin{aligned}
p_{2}= & \beta^{2}\left\{\frac{c_{22}\left(-c_{12}^{2}+4 c_{66}^{2}+c_{11} c_{22}\right) n^{2}\left(n^{2}-1\right)^{2}}{12\left(c_{12}^{2}-c_{11} c_{22}\right) c_{66}}\right. \\
& \left.+c_{11} \Omega^{2} \frac{2 c_{12} c_{66} n^{2}+c_{12}^{2}\left(n^{2}+1\right)-c_{22}\left(c_{11} n^{2}+c_{11}+c_{66}\right)}{\left(c_{12}^{2}-c_{11} c_{22}\right) c_{66}}\right\}+\ldots
\end{aligned}
$$

and

$$
\begin{aligned}
q_{2}= & \beta^{2}\left(\frac{c_{11} c_{22} n^{2}\left(n^{2}+1\right) \Omega^{2}}{c_{12}^{2}-c_{11} c_{22}}-\frac{c_{22}^{2} n^{4}\left(n^{2}-1\right)^{2}}{12\left(c_{12}^{2}-c_{11} c_{22}\right)}\right)+\frac{\beta^{4}}{\left(c_{12}^{2}-c_{11} c_{22}\right)^{2} c_{66}} \times \\
& \left\{c_{11}^{2} \Omega^{4}\left[c_{11} c_{22}\left(-c_{66} n^{4}+c_{22} n^{2}+c_{22}\right)-c_{12}^{2}\left(\left(c_{22}+c_{66}\right) n^{2}+c_{22}\right)\right]\right. \\
& +\frac{1}{72} c_{22}^{2}\left(n^{2}-1\right)^{2} n^{4}\left[c_{12}^{3} n^{2}\left(n^{2}-1\right)+c_{66} c_{12}^{2}\left(1-2 n^{2}\right)^{2}\right. \\
& \left.-\left(c_{11} c_{22}-4 c_{66}^{2}\right) c_{12} n^{2}\left(n^{2}-1\right)+c_{11} c_{22} c_{66}\left(-3 n^{4}+3 n^{2}-1\right)\right] \\
+ & \frac{1}{12} n^{2} \Omega^{2} c_{11} c_{22}\left[-2 c_{12}^{3} n^{2}\left(n^{4}-1\right)+2\left(c_{11} c_{22}-4 c_{66}^{2}\right) c_{12} n^{2}\left(n^{4}-1\right)\right. \\
& +c_{12}^{2}\left(c_{22}\left(n^{2}-1\right)^{2}+c_{66}\left(-8 n^{6}+n^{4}+7 n^{2}-2\right)\right) \\
& \left.\left.-c_{11} c_{22}\left(c_{22}\left(n^{2}-1\right)^{2}+c_{66}\left(-7 n^{6}+3 n^{4}+4 n^{2}-2\right)\right)\right]\right\}+\ldots
\end{aligned}
$$

In the expansion for $q_{2}$ we keep two terms due to the possibility of degeneration near the cutoff frequency $\Omega=\Omega_{*}$ given by the equation $q_{2}=0$. Indeed, on introducing the asymptotic expansion

$$
\Omega_{*}^{2}=\Omega_{0}^{2}+\beta^{2} \Omega_{1}^{2}+\ldots
$$

and upon solving $q_{2}=0$, we find, at leading order,

$$
\Omega_{0}^{2}=\frac{c_{22} n^{2}\left(n^{2}-1\right)^{2}}{12 c_{11}\left(n^{2}+1\right)},
$$

while, proceeding to the next order, we get the correction

$$
\Omega_{1}^{2}=-\frac{c_{22} n^{6}\left(n^{2}-1\right)^{2}}{36 c_{11}\left(n^{2}+1\right)^{3}}
$$

Consequently, we may write

$$
q_{2}=-\beta^{2} \frac{c_{11} c_{22} n^{2}\left(n^{2}+1\right)}{c_{11} c_{22}-c_{12}^{2}}\left(\Omega^{2}-\Omega_{*}^{2}\right)+\ldots
$$




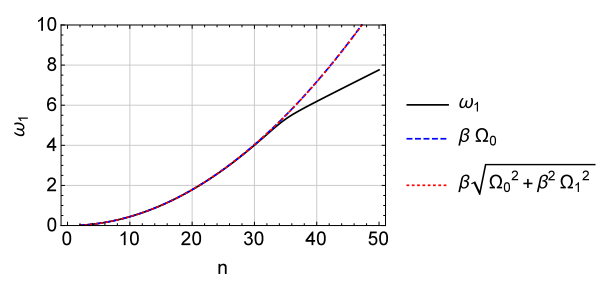

Figure 3: Kirchhoff-Love (solid) vs. one-term (dashed) and two-term (dotted) asymptotic expression for the cutoff frequency $\omega_{1}$ for a graphite-epoxy composite shell (parameters as in $(8)$ and $\beta=0.1$ ) vs. circular wavenumber $n$. The two-term expansion coincides with the one-term expansion for this parameter set and it well approximates the exact expression (16) up to $n=35$
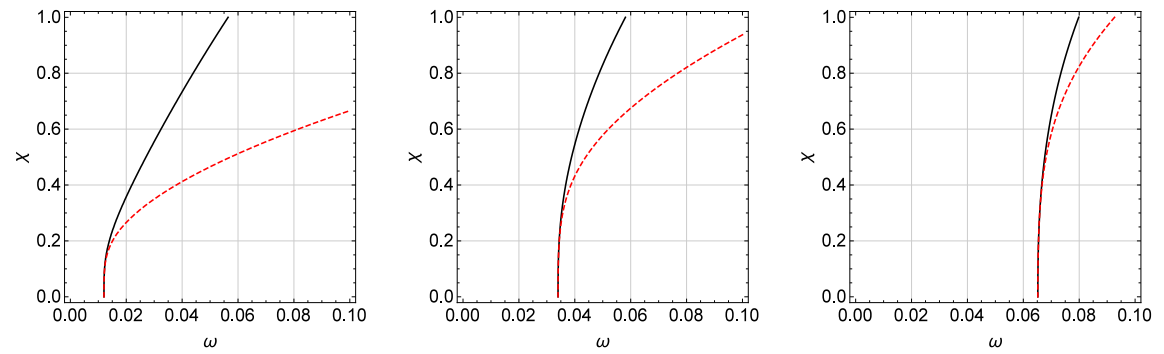

Figure 4: Kirchhoff-Love (solid) vs. shortened (dashed) dispersion relation for a graphiteepoxy composite shell for three different circular wavenumbers (parameters as in (8) $\beta=0.1$ and, from left to right, $n=2,3,4)$

Thus, the lowest cutoff frequency has the asymptotic expansion

$$
\omega_{*}^{2} \equiv \omega_{1}^{2}=\beta^{2} \Omega_{0}^{2}+\beta^{4} \Omega_{1}^{2}+\cdots,
$$

which, as shown in Fig.3, well approximates the exact formula (16).

The sought-for shortened dispersion relation reads

$$
\chi^{4}-p_{2} \chi^{2}+q_{2}=0
$$

and, at leading order, it can be simplified to

$$
\chi^{4}+q_{2}=0
$$

whenever $\Omega-\Omega_{*} \gg \beta^{2}$. Likewise, in the close vicinity of the cutoff frequency $\left(\Omega-\Omega_{*} \ll \beta^{2}\right)$, it may be reduced to

$$
\chi^{2}-p_{2}=0
$$



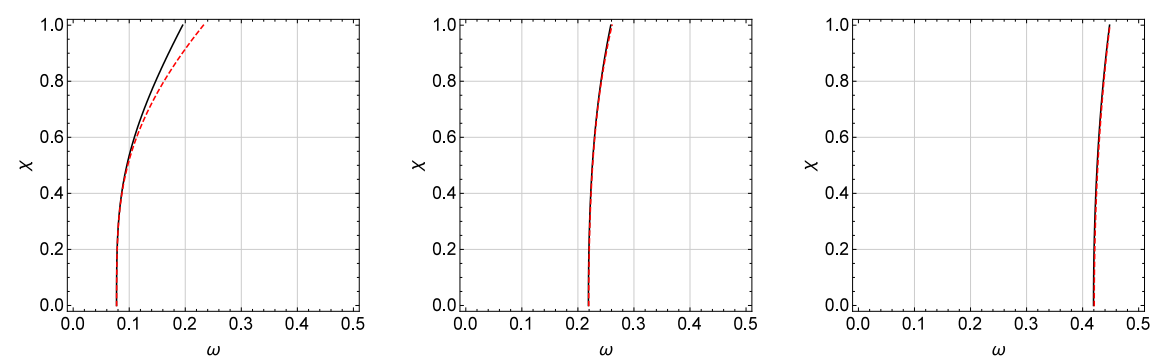

Figure 5: Kirchhoff-Love (solid) vs. shortened (dashed) dispersion relation for an isotropic shell $(\nu=0.3, \beta=0.1$ and, from left to right, $n=2,3,4)$
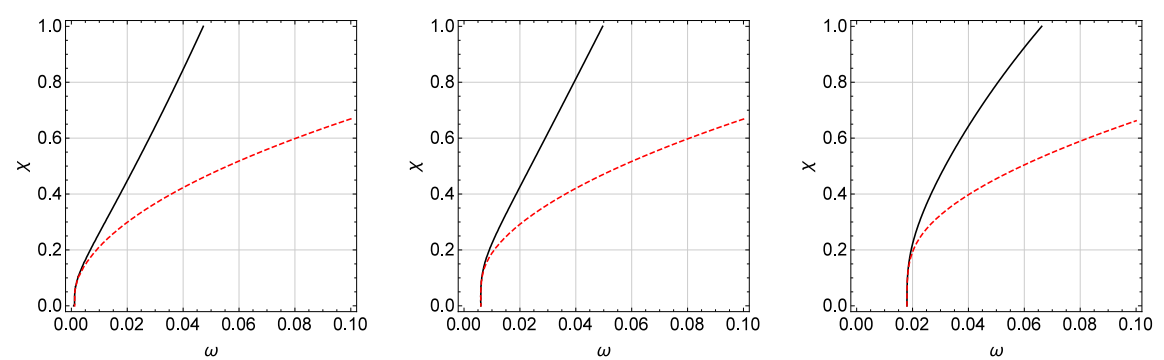

Figure 6: Kirchhoff-Love (solid) vs. shortened (dashed) dispersion relation for a graphiteepoxy composite shell for three different values of the relative thickness $\beta$ (parameters as in (8) $n=2$ and, from left to right, $\beta=0.01,0.05,0.15$ )

As a consequence, the term $p_{2}$ may be further simplified by assuming $\Omega^{2}=\Omega_{0}^{2}$

$$
p_{2}=\beta^{2} \frac{c_{22} n^{2}\left(n^{2}-1\right)^{2}\left(c_{22}-2\left(c_{12} n^{2}+2 c_{66}\left(n^{2}+1\right)\right)\right)}{12\left(c_{11} c_{22}-c_{12}^{2}\right)\left(n^{2}+1\right)},
$$

since its role is essential only inasmuch as $\Omega-\Omega_{0} \lesssim \beta^{2}$. Then, the shortened dispersion relation may be rewritten in the form

$$
\gamma \chi^{4}+\delta \beta^{2} \chi^{2}+\omega_{*}^{2}-\omega^{2}=0
$$

where we have let the positive quantities

$$
\gamma=\frac{c_{11} c_{22}-c_{12}^{2}}{c_{11} c_{22} n^{2}\left(n^{2}+1\right)}
$$

and

$$
\delta=-p_{2} \gamma=\frac{\left(n^{2}-1\right)^{2}\left[2 c_{12} n^{2}+4 c_{66}\left(n^{2}+1\right)-c_{22}\right]}{12 c_{11}\left(n^{2}+1\right)^{2}} .
$$

In the isotropic case, see Eqs.(9), we have

$$
\delta_{i s o}=\frac{\left(1-n^{2}\right)^{2}\left(2 n^{2}+1-2 \nu\right)}{12\left(1+n^{2}\right)^{2}}, \quad \gamma_{i s o}=\frac{1-\nu^{2}}{n^{2}\left(1+n^{2}\right)},
$$


and

$$
\Omega_{0 \text { iso }}^{2}=\frac{n^{2}\left(1-n^{2}\right)^{2}}{12\left(1+n^{2}\right)}, \quad \Omega_{1 \text { iso }}^{2}=\frac{n^{6}\left(1-n^{2}\right)^{2}}{36\left(1+n^{2}\right)^{3}},
$$

which correspond to the analogous results given in [9]. Fig.4 plots the dispersion curve for the lowest vibration mode, as given by Eq.(14), along with its approximation (30) for graphite-epoxy composite material at $n=2,3$ and 4 . For comparison, Fig.5 presents similar results for an isotropic material. Clearly, the quality of the approximation is reduced in the orthotropic case owing to large ratios between the elastic constants for graphite-epoxy composite material. Finally, Fig.6 illustrates the effect of the relative thickness $\beta$ on the accuracy of the derived approximation.

\section{Refined semi-membrane theory}

Consideration of long-wave low-frequency behaviour, i.e. $\chi \ll 1$ and $\omega \ll 1$, yields the following approximate expressions for the eigenmodes (B.1,B.2)

$$
\begin{gathered}
U_{n}=\left[-\frac{1}{n^{2}}+\beta^{2}\left(-\frac{c_{11}\left(c_{12}+c_{22}+c_{66}\right) \Omega^{2}}{c_{22} c_{66} n^{4}}+\frac{\left(c_{12}+2 c_{66}\right)\left(n^{2}-1\right)}{12 c_{66} n^{2}}\right)\right] \imath \chi W_{n} \\
+\frac{c_{11} c_{22}-c_{12}^{2}-c_{66} c_{12}}{c_{22} c_{66} n^{4}} \imath \chi^{3} W_{n}+\ldots
\end{gathered}
$$

and

$$
V_{n}=-\frac{1}{n} W_{n}-\frac{c_{12}}{c_{22} n^{3}} \chi^{2} W_{n}-\left(\frac{c_{11}}{c_{22} n^{3}} \Omega^{2}+\frac{n^{2}-1}{12 n}\right) \beta^{2} W_{n}+\ldots
$$

Let us adapt in the last formulae the operatorial identities

$$
\left(\imath \alpha^{-1} \chi\right)^{j}\left\{U_{n}, V_{n}, W_{n}\right\}=\partial_{\xi^{j}}^{j}\left\{u_{n}, v_{n}, w_{n}\right\}, \quad j \in \mathbb{N},
$$

and

$$
\Omega^{2}=-\partial_{T T}^{2}
$$

This gives

$$
v_{n}+\frac{1}{n} w_{n}=\frac{c_{12}}{c_{22} n^{3}} \alpha^{2} \partial_{\xi \xi}^{2} w_{n}+\frac{c_{11}}{c_{22} n^{3}} \beta^{2} \partial_{T T}^{2} w_{n}-\beta^{2} \frac{n^{2}-1}{12 n} w_{n}
$$


and

$$
\begin{aligned}
u_{n}+ & n^{-2} \alpha \partial_{\xi} w_{n}=\frac{c_{11}\left(c_{12}+c_{22}+c_{66}\right)}{c_{22} c_{66} n^{4}} \alpha \beta^{2} \partial_{\xi T T}^{3} w_{n} \\
& +\frac{\left(c_{12}+2 c_{66}\right)\left(n^{2}-1\right)}{12 c_{66} n^{2}} \alpha \beta^{2} \partial_{\xi} w_{n}-\frac{c_{11} c_{22}-c_{12}^{2}-c_{66} c_{12}}{c_{22} c_{66} n^{4}} \alpha^{3} \partial_{\xi \xi \xi}^{3} w_{n},
\end{aligned}
$$

thus refining at $\alpha \ll 1$ and $\beta \ll 1$, the well-known geometric assumptions of the semi-membrane theory expressing the vanishing of the mid-surface deformation due to shear and circumferential elongation [7]

$$
v_{n}+n^{-1} w_{n}=0, \quad \text { and } \quad u_{n}+\alpha n^{-2} \frac{\mathrm{d} w_{n}}{\mathrm{~d} \xi}=0 .
$$

It is well-known that, in statics and in dynamics, outside the narrow vicinity of the cutoff frequency, the semi-membrane theory is asymptotically consistent $[7,8]$.

Introducing the refined assumptions Eqs. $(35,36)$ in the Lagrangian density (1), we may write a reduced variational principle $[6,27]$

$$
\int_{T_{1}}^{T_{2}} \int_{0}^{L} \mathcal{L}_{0} \mathrm{~d} \xi \mathrm{d} T
$$

where the Lagrangian density is expanded to within $O\left[\left(\alpha^{2}+\beta^{2}\right)^{2}\right]$

$$
\begin{aligned}
& \mathcal{L}_{0}= \beta^{2}\left(\partial_{T} w_{n}\right)^{2}+\beta^{2} \frac{c_{22} n^{2}\left(n^{2}-1\right)^{2}\left(-\beta^{2}+3\left(\beta^{2}-4\right) n^{2}-12\right)}{144 c_{11}\left(n^{2}+1\right)^{2}} w_{n}^{2} \\
&-\alpha^{2} \beta^{2} \frac{c_{66}\left(n^{2}-1\right)^{2}}{3 c_{11}\left(n^{2}+1\right)}\left(\partial_{\xi} w_{n}\right)^{2}+\alpha^{2} \beta^{2} \frac{c_{12}\left(n^{2}-1\right)^{2}}{6 c_{11}\left(n^{2}+1\right)} w_{n} \partial_{\xi \xi}^{2} w_{n} \\
&+\alpha^{4} \frac{c_{12}^{2}-c_{11} c_{22}}{c_{11} c_{22} n^{2}\left(n^{2}+1\right)}\left(\partial_{\xi \xi}^{2} w_{n}\right)^{2}-\alpha^{2} \beta^{2} \frac{2 c_{12}}{c_{22} n^{2}\left(n^{2}+1\right)} \partial_{T} w_{n} \partial_{\xi \xi T}^{3} w_{n} \\
&-\beta^{4} \frac{c_{11}}{c_{22} n^{2}\left(n^{2}+1\right)}\left(\partial_{T T}^{2} w_{n}\right)^{2}-\beta^{4} \frac{2 c_{11}}{c_{22} n^{2}\left(n^{2}+1\right)} \partial_{T} w_{n} \partial_{T T T}^{3} w_{n}+\alpha^{2} \beta^{2} \frac{1}{n^{2}\left(n^{2}+1\right)}\left(\partial_{\xi T}^{2} w_{n}\right)^{2}
\end{aligned}
$$

Performing the variation with respect to $w_{n}$, we get the PDE

$$
\begin{gathered}
\alpha^{4} \frac{c_{11} c_{22}-c_{12}^{2}}{c_{11} c_{22} n^{2}\left(n^{2}+1\right)} \partial_{\xi \xi \xi \xi}^{4} w_{n}-\beta^{4} \frac{c_{11}}{c_{22} n^{2}\left(n^{2}+1\right)} \partial_{T T T T}^{4} w_{n}+\beta^{2} \partial_{T T}^{2} w_{n} \\
-\alpha^{2} \beta^{2} \frac{\left(c_{12}+2 c_{66}\right)\left(n^{2}-1\right)^{2}}{6 c_{11}\left(n^{2}+1\right)} \partial_{\xi \xi}^{2} w_{n}-\alpha^{2} \beta^{2} \frac{2 c_{12}+c_{22}}{c_{22} n^{2}\left(n^{2}+1\right)} \partial_{\xi \xi T T}^{4} w_{n} \\
-\beta^{2} \frac{c_{22} n^{2}\left(n^{2}-1\right)^{2}\left(-\beta^{2}+3\left(\beta^{2}-4\right) n^{2}-12\right)}{144 c_{11}\left(n^{2}+1\right)^{2}} w_{n}=0
\end{gathered}
$$


whose dispersion relation is asymptotically equivalent to Eq.(30), as it follows from the discussion at the end of the previous section, see Eq.(29). Indeed, we can simplify Eq.(38) through setting

$$
\partial_{T T T T}^{4} w_{n}=\Omega_{0}^{4} w_{n}, \quad \partial_{\xi \xi T T}^{4} w_{n}=-\Omega_{0}^{2} \partial_{\xi \xi}^{2} w_{n}
$$

to get

$$
\gamma \alpha^{4} \partial_{\xi \xi \xi \xi}^{4} w_{n}-\delta \alpha^{2} \beta^{2} \partial_{\xi \xi}^{2} w_{n}+\beta^{2} \partial_{T T}^{2} w_{n}+\omega_{*}^{2} w_{n}=0 .
$$

It is worth noting that the conventional semi-membrane hypotheses, Eqs.(37), generally do not result in Eq.(39), see [9] for further detail. In the original variables $x$ and $t$, the last equation reads as

$$
\gamma R^{4} \partial_{x x x x}^{4} w_{n}-\delta h^{2} \partial_{x x}^{2} w_{n}+\left(\frac{R}{c_{L}}\right)^{2} \partial_{t t}^{2} w_{n}+\left(\frac{h}{R}\right)^{2} \Omega_{*}^{2} w_{n}=0
$$

which corresponds to the governing equation of a beam supported by an elastic two-parameter (Pasternak-type) foundation, e.g. see [28, 29]. It is worth noting that in the standard semi-membrane equation [8, Eq.(20)] the term with the second derivative with respect to $x$ does not appear and also $\Omega_{*}=\Omega_{0}$.

\section{Concluding remarks}

This paper presents a low-frequency analysis of the propagating modes in an orthotropic cylindrical shell. The investigation is partly motivated by recent experimental findings indicating that carbon and oxidic nanotubes possess strong anisotropic material properties.

The developed approach is based on the observation that the lowest propagating vibration modes in an orthotropic cylindrical shell are very sensitive to the variation of the problem's parameters, in contrast to the complementary evanescent modes, which, at leading order, are independent of frequency and circumferential wavenumber. Within the framework of the Kirchhoff-Love theory, the propagating and the evanescent modes correspond to the semi-membrane and to the edge effect approximations, respectively [7]. Although, for a number of practically important set-ups, the highly localised edge effect appears 
of limited interest, it is here put to advantage for the extraction of the propagating modes. Indeed, the shortened dispersion equation for the latter comes immediately through polynomial division of the full Kirchhoff-Love dispersion equation by the relatively straightforward equation governing the evanescent modes. Similarly to the isotropic case [9], this shortened equation may be regarded as the leading order expansion near the lowest cutoff frequency. Besides, it is shown that the same equation also follows through a refined dynamic version of the semi-membrane shell theory obtained by a variational procedure. Amended physical assumptions underlying this theory are also formulated. $\mathrm{Nu}$ merical results show that the shortened dispersion equation provides a very robust approximation of the bending vibration branch.

The presented approach has a potential to be extended to other types of anisotropy. In addition, the proposed dynamic semi-membrane model may be generalised to incorporating the effect of weak non-linearity.

\section{Acknowledgments}

JK gratefully acknowledges a visiting professor fellowship by the University of Modena and Reggio Emilia under the Short Staying Fellowship Programme 2016. 


\section{Appendix A. Coefficients in the dispersion relation}

The coefficients in Eq.(14) are

$$
\begin{aligned}
a_{0} & =6912 \beta^{-2} \omega^{2}\left(c_{11}^{2} \omega^{2}\left(c_{22} n^{2}+c_{66} n^{2}+c_{22}\right)-c_{11} c_{22} c_{66} n^{2}\left(n^{2}+1\right)-c_{11}^{3} \omega^{4}\right) \\
& +144 c_{11}^{2} n^{2} \omega^{4}\left(4 c_{22}\left(n^{2}+1\right)+c_{66}\right) \\
& +12 \beta^{2}\left(c_{22}^{2} c_{66} n^{4}\left(n^{2}-1\right)^{2}-c_{11} c_{22} c_{66} n^{4}\left(n^{2}+1\right) \omega^{2}\right) \\
& -144 c_{11} c_{22} n^{2} \omega^{2}\left(4 c_{22}\left(n^{2}-1\right)^{2}+c_{66}\left(4 n^{4}+5 n^{2}+1\right)\right)+576 c_{22}^{2} c_{66}\left(n^{2}-1\right)^{2} n^{4} \\
a_{1} & =-12 \beta^{2} c_{11}\left(2 c_{12}+c_{22}\right) c_{66} n^{4} \omega^{2} \\
& +6912 \beta^{-2} \omega^{2}\left(c_{11}^{2}\left(c_{11}+c_{66}\right) \omega^{2}-c_{11}\left(-2 c_{12} c_{66} n^{2}-c_{12}^{2}\left(n^{2}+1\right)+c_{22}\left(c_{11} n^{2}+c_{11}+c_{66}\right)\right)\right) \\
& +144 c_{11}^{2} \omega^{4}\left(8 c_{12} n^{2}+c_{66}\left(16 n^{2}+9\right)\right)+576 c_{22}\left(-c_{12}^{2}+4 c_{66}^{2}+c_{11} c_{22}\right)\left(n^{2}-1\right)^{2} n^{2} \\
& +144 c_{11} \omega^{2}\left(-2 c_{66} n^{2}\left(c_{12}\left(4 n^{2}+7\right)+8 c_{66}\left(n^{2}+1\right)\right)\right. \\
& \left.-c_{22}\left(4 n^{2}\left(c_{11} n^{2}+2 c_{12} n^{2}+c_{11}-2 c_{12}\right)+c_{66}\left(20 n^{4}-24 n^{2}+9\right)\right)\right) \\
a_{2} & =\beta^{6}\left(c_{11} c_{22}-c_{12}^{2}\right) c_{66} n^{4} \\
& +\beta^{4}\left(72 c_{66} n^{2}\left(c_{11} c_{22} n^{2}-c_{12}^{2}\right)-12 c_{11}\left(-4 c_{12}^{2}-6 c_{66} c_{12}+c_{11}\left(4 c_{22}+c_{66}\right)\right) n^{2} \omega^{2}\right) \\
& +\beta^{2}\left(-144 c_{11} \omega^{2}\left(8 c_{66}\left(c_{12}+2 c_{66}\right) n^{2}+c_{11}\left(4\left(2 c_{12}+c_{22}\right) n^{2}+c_{66}\left(20 n^{2}+9\right)\right)\right)\right. \\
& +144\left(-8 c_{12}^{3} n^{2}\left(n^{2}-1\right)+8\left(c_{11} c_{22}-4 c_{66}^{2}\right) c_{12} n^{2}\left(n^{2}-1\right)\right. \\
& \left.\left.+c_{66} c_{12}^{2}\left(-32 n^{4}+32 n^{2}-9\right)+3 c_{11} c_{22} c_{66}\left(8 n^{4}-8 n^{2}+3\right)\right)+576 c_{11}^{3} \omega^{4}\right) \\
& -6912 c_{11}^{2} c_{66} \omega^{2}+6912 c_{66}\left(c_{11} c_{22}-c_{12}^{2}\right) \\
a_{3} & =\beta^{2}\left(48 c_{11}\left(-c_{12}^{2}+4 c_{66}^{2}+c_{11} c_{22}\right) n^{2}-108 c_{11}^{2} c_{66} \omega^{2}\right) \\
& +576 c_{11}\left(-c_{12}^{2}+4 c_{66}^{2}+c_{11} c_{22}\right) n^{2}-576 c_{11}^{2}\left(c_{11}+c_{66}\right) \omega^{2} \\
a_{4} & =108 \beta^{2} c_{66} c_{11}^{2}+576 c_{66} c_{11}^{2}
\end{aligned}
$$


The coefficients in the expansion (19) are

$$
\begin{aligned}
& a_{4}^{(0)}=576 c_{11}^{2} c_{66}, \\
& a_{4}^{(2)}=108 c_{11}^{2} c_{66} \\
& a_{3}^{(0)}=576 c_{11}\left(-c_{12}^{2}+4 c_{66}^{2}+c_{11} c_{22}\right) n^{2}, \\
& a_{3}^{(2)}=-576 c_{11}^{2}\left(c_{11}+c_{66}\right) \Omega^{2}+48 c_{11}\left(-c_{12}^{2}+4 c_{66}^{2}+c_{11} c_{22}\right) n^{2}, \\
& a_{3}^{(4)}=-108 c_{11}^{2} c_{66} \Omega^{2} \\
& a_{2}^{(0)}=6912 c_{66}\left(c_{11} c_{22}-c_{12}^{2}\right) \text {, } \\
& a_{2}^{(2)}=144\left(-8 c_{12}^{3} n^{2}\left(n^{2}-1\right)+8\left(c_{11} c_{22}-4 c_{66}^{2}\right) c_{12} n^{2}\left(n^{2}-1\right)\right. \\
& \left.+c_{66} c_{12}^{2}\left(-32 n^{4}+32 n^{2}-9\right)+3 c_{11} c_{22} c_{66}\left(8 n^{4}-8 n^{2}+3\right)\right)-6912 c_{11}^{2} c_{66} \Omega^{2}, \\
& a_{2}^{(4)}=72 c_{66} n^{2}\left(c_{11} c_{22} n^{2}-c_{12}^{2}\right)-144 c_{11} \Omega^{2}\left(8 c_{66}\left(c_{12}+2 c_{66}\right) n^{2}\right. \\
& \left.+c_{11}\left(4\left(2 c_{12}+c_{22}\right) n^{2}+c_{66}\left(20 n^{2}+9\right)\right)\right) \\
& a_{2}^{(6)}=\left(c_{11} c_{22}-c_{12}^{2}\right) c_{66} n^{4}-12 c_{11}\left(-4 c_{12}^{2}-6 c_{66} c_{12}+c_{11}\left(4 c_{22}+c_{66}\right)\right) n^{2} \Omega^{2}+576 c_{11}^{3} \Omega^{4} \\
& a_{1}^{(0)}=-6912 c_{11} \Omega^{2}\left(-2 c_{12} c_{66} n^{2}-c_{12}^{2}\left(n^{2}+1\right)+c_{22}\left(c_{11} n^{2}+c_{11}+c_{66}\right)\right) \\
& -576 c_{22}\left(c_{12}^{2}-4 c_{66}^{2}-c_{11} c_{22}\right) n^{2}\left(n^{2}-1\right)^{2}, \\
& a_{1}^{(2)}=-144 c_{11} \Omega^{2}\left(2 c_{66} n^{2}\left(c_{12}\left(4 n^{2}+7\right)+8 c_{66}\left(n^{2}+1\right)\right)\right. \\
& \left.+c_{22}\left(4 n^{2}\left(c_{11} n^{2}+2 c_{12} n^{2}+c_{11}-2 c_{12}\right)+c_{66}\left(20 n^{4}-24 n^{2}+9\right)\right)\right)+6912 c_{11}^{2}\left(c_{11}+c_{66}\right) \Omega^{4}, \\
& a_{1}^{(4)}=-12 c_{11}\left(2 c_{12}+c_{22}\right) c_{66} n^{4} \Omega^{2}+144 c_{11}^{2} \Omega^{4}\left(8 c_{12} n^{2}+c_{66}\left(16 n^{2}+9\right)\right), \\
& a_{0}^{(0)}=576 c_{22}^{2} c_{66} n^{4}\left(n^{2}-1\right)^{2}-6912 c_{11} c_{22} c_{66} n^{2}\left(n^{2}+1\right) \Omega^{2} \text {, } \\
& a_{0}^{(2)}=6912 c_{11}^{2} \Omega^{4}\left(c_{22} n^{2}+c_{66} n^{2}+c_{22}\right)-144 c_{11} c_{22} n^{2} \Omega^{2}\left(4 c_{22}\left(n^{2}-1\right)^{2}+c_{66}\left(4 n^{4}+5 n^{2}+1\right)\right) \\
& +12 c_{22}^{2} c_{66}\left(n^{2}-1\right)^{2} n^{4}, \\
& a_{0}^{(4)}=144 c_{11}^{2} n^{2} \Omega^{4}\left(4 c_{22}\left(n^{2}+1\right)+c_{66}\right)-12 c_{11} c_{22} c_{66} n^{4}\left(n^{2}+1\right) \Omega^{2}-6912 c_{11}^{3} \Omega^{6}, \\
& \text { and } a_{4}^{(4)}=a_{4}^{(6)}=a_{3}^{(6)}=a_{1}^{(6)}=a_{0}^{(6)}=0 \text {. }
\end{aligned}
$$




\section{Appendix B. Eigenforms}

The expressions for the eigenforms are

$$
\begin{gathered}
U_{n}=\left\{4 \imath \chi W _ { 1 } \left[3 n^{2}\left(16\left(c_{12}+c_{66}\right)-\beta^{2} c_{66}\right)\left(\beta^{2}\left(c_{12}+3 c_{66}\right) \chi^{2}+c_{22}\left(\beta^{2} n^{2}+12\right)\right)\right.\right. \\
\left.\left.-\left(12 c_{12}-\beta^{2} c_{66} n^{2}\right)\left(3\left(3 \beta^{2}+16\right) c_{66} \chi^{2}+4\left(\beta^{2}+12\right) c_{22} n^{2}-48 c_{11} \omega^{2}\right)\right]\right\} / \\
\left\{9 n^{2} \chi^{2}\left(\beta^{2} c_{66}-16\left(c_{12}+c_{66}\right)\right)^{2}-\left(48 c_{11}\left(\omega^{2}-\chi^{2}\right)-\left(\beta^{2}+48\right) c_{66} n^{2}\right) \times\right. \\
\left.\left(-3\left(3 \beta^{2}+16\right) c_{66} \chi^{2}-4\left(\beta^{2}+12\right) c_{22} n^{2}+48 c_{11} \omega^{2}\right)\right\}
\end{gathered}
$$

and

$$
\begin{aligned}
& V_{n}=\{ n W_{1}\left[\chi ^ { 2 } \left(48 \beta^{2} c_{66}\left(4 c_{66} n^{2}+3 c_{11}\left(\chi^{2}-\omega^{2}\right)\right)\right.\right. \\
&+c_{12}(\left.\left.48 \beta^{2} c_{11}\left(\chi^{2}-\omega^{2}\right)+c_{66}\left(\beta^{2}\left(\left(\beta^{2}+96\right) n^{2}+36\right)-576\right)\right)-576 c_{12}^{2}\right) \\
&+\left.\left.c_{22}\left(\beta^{2} n^{2}+12\right)\left(\left(\beta^{2}+48\right) c_{66} n^{2}+48 c_{11}\left(\chi^{2}-\omega^{2}\right)\right)\right]\right\} / \\
&\left\{\left(\beta^{2}+12\right) c_{22} n^{2}\left(\left(\beta^{2}+48\right) c_{66} n^{2}+48 c_{11}\left(\chi^{2}-\omega^{2}\right)\right)\right. \\
&+12\left[3\left(3 \beta^{2}+16\right) c_{11} c_{66} \chi^{4}-c_{11} \omega^{2}\left(3 \chi^{2}\left(3 \beta^{2} c_{66}+16\left(c_{11}+c_{66}\right)\right)+\left(\beta^{2}+48\right) c_{66} n^{2}\right)\right. \\
&\left.\left.+2 n^{2} \chi^{2}\left(\beta^{2} c_{66}\left(3 c_{12}+8 c_{66}\right)-24 c_{12}\left(c_{12}+2 c_{66}\right)\right)+48 c_{11}^{2} \omega^{4}\right]\right\} \quad(B .2)
\end{aligned}
$$

\section{References}

[1] C. Li and T. Chou, "Single-walled carbon nanotubes as ultrahigh frequency nanomechanical resonators," Physical review B, vol. 68, no. 7, p. 073405, 2003.

[2] V. Sazonova, Y. Yaish, H. Üstünel, D. Roundy, T. A. Arias, and P. L. McEuen, "A tunable carbon nanotube electromechanical oscillator," $\mathrm{Na}$ ture, vol. 431, no. 7006, pp. 284-287, 2004.

[3] H. Peng, C. Chang, S. Aloni, T. Yuzvinsky, and A. Zettl, "Ultrahigh frequency nanotube resonators," Physical Review Letters, vol. 97, no. 8, p. $087203,2006$. 
[4] M. Anantram and F. Leonard, "Physics of carbon nanotube electronic devices," Reports on Progress in Physics, vol. 69, no. 3, p. 507, 2006.

[5] R. R. Hartmann, J. Kono, and M. E. Portnoi, "Terahertz science and technology of carbon nanomaterials," Nanotechnology, vol. 25, no. 32, p. 322001, 2014.

[6] M. Strozzi, L. I. Manevitch, F. Pellicano, V. V. Smirnov, and D. S. Shepelev, "Low-frequency linear vibrations of single-walled carbon nanotubes: Analytical and numerical models," Journal of Sound and Vibration, vol. 333, no. 13, pp. 2936-2957, 2014.

[7] A. L. Gol'Denveizer, Theory of Elastic Thin Shells: Solid and Structural Mechanics. Pergamon Press, Oxford, UK, 1961.

[8] J. Kaplunov, L. Kossovich, and M. Wilde, "Free localized vibrations of a semi-infinite cylindrical shell," The Journal of the Acoustical Society of America, vol. 107, no. 3, pp. 1383-1393, 2000.

[9] J. Kaplunov, L. Manevitch, and V. Smirnov, "Vibrations of an elastic cylindrical shell near the lowest cut-off frequency," in Proc. R. Soc. A, vol. 472, p. 20150753, The Royal Society, 2016.

[10] J.-P. Salvetat, A. J. Kulik, J.-M. Bonard, G. A. D. Briggs, T. Stöckli, K. Méténier, S. Bonnamy, F. Béguin, N. A. Burnham, and L. Forró, "Elastic modulus of ordered and disordered multiwalled carbon nanotubes," Advanced Materials, vol. 11, no. 2, pp. 161-165, 1999.

[11] O. Blakslee, D. Proctor, E. Seldin, G. Spence, and T. Weng, "Elastic constants of compression-annealed pyrolytic graphite," Journal of Applied Physics, vol. 41, no. 8, pp. 3373-3382, 1970.

[12] J. Lu, "Elastic properties of carbon nanotubes and nanoropes," Physical Review Letters, vol. 79, no. 7, p. 1297, 1997. 
[13] T. Chang, "A molecular based anisotropic shell model for single-walled carbon nanotubes," Journal of the Mechanics and Physics of Solids, vol. 58, no. 9, pp. 1422-1433, 2010.

[14] S. Fazelzadeh and E. Ghavanloo, "Nonlocal anisotropic elastic shell model for vibrations of single-walled carbon nanotubes with arbitrary chirality," Composite Structures, vol. 94, no. 3, pp. 1016-1022, 2012.

[15] G. Patzke, F. Krumeich, and R. Nesper, "Oxidic nanotubes and nanorodsanisotropic modules for a future nanotechnology," Angewandte Chemie International Edition, vol. 41, no. 14, pp. 2446-2461, 2002.

[16] A. Love, A treatise on the mathematical theory of elasticity. Dover Publications, Inc., New York, USA, 1944.

[17] J. Kaplunov, L. Y. Kossovich, and G. Rogerson, "Direct asymptotic integration of the equations of transversely isotropic elasticity for a plate near cut-off frequencies," The Quarterly Journal of Mechanics and Applied Mathematics, vol. 53, no. 2, pp. 323-341, 2000.

[18] J. Kaplunov and D. Markushevich, "Plane vibrations and radiation of an elastic layer lying on a liquid half-space," Wave Motion, vol. 17, no. 3, pp. 199-211, 1993.

[19] J. Kaplunov and E. Nolde, "Long-wave vibrations of a nearly incompressible isotropic plate with fixed faces," The Quarterly Journal of Mechanics and Applied Mathematics, vol. 55, no. 3, pp. 345-356, 2002.

[20] M. Amabili, Nonlinear vibrations and stability of shells and plates. Cambridge University Press, Cambridge, UK, 2008.

[21] W. Flügge, Stresses in shells. Springer, Berlin, DL, second ed., 1973.

[22] R. M. Jones, Mechanics of composite materials, vol. 193. Taylor Francis, Philadelphia, USA, 1999.

[23] M. J. Musgrave, Crystal acoustics. Holden-Day, San Francisco, 1970. 
[24] A. Kleshchev, "Against phase veloities of elastic waves in thin transversely isotropic cylindrical shell," Open Journal of Acoustics, vol. 3, no. 03, p. 67, 2013.

[25] C. Chapman and S. Sorokin, "The deferred limit method for long waves in a curved waveguide," in Proc. R. Soc. A, The Royal Society, 2017. to appear.

[26] J. R. Vinson, The behavior of thin walled structures: beams, plates, and shells. Kluwer Academic Publishers, Boston, USA, 1921.

[27] M. Destrade, Y. Fu, and A. Nobili, "Edge wrinkling in elastically supported pre-stressed incompressible isotropic plates," in Proc. R. Soc. A, vol. 472, p. 20160410, The Royal Society, 2016.

[28] A. Nobili, "Variational approach to beams resting on two-parameter tensionless elastic foundations," Journal of Applied Mechanics, vol. 79, no. 2, p. 021010, 2012.

[29] J. Kaplunov and A. Nobili, "The edge waves on a Kirchhoff plate bilaterally supported by a two-parameter elastic foundation," Journal of Vibration and Control, p. 1077546315606838, 2015. 